\title{
DESCRIPCION DE LA PUPA DE CULEX (ANOEDIOPORPA) BAMBORUM ROZEBOOM Y KOMP 1948 (DIPTERA: CULICIDAE)
}

\author{
ALBERTO MORALES. * EMILIO CURA. **
}

\begin{abstract}
Se describe la pupa de Culex (Anoedioporpa) bamborum a partir de material procedente de una colonia de esta especie de mosquito establecida en el insectario del INS en el municipio de Armero (Tolima), Colombia, S.A.
\end{abstract}

\section{INTRODUCCION}

En el año de 1.948, Rozeboom y Komp describieron el adulto y la larva de Culex (Anoedioporpa) bamburum (1)

La especie fue colocada inicialmente en el subgénero Tinolestes; en 1965 Belkin y col. (2) en sus estudios sobre mosquitos de América la incluyeron en el subgénero Aedinus pero en 1.977 Kenneth L. Knight y Alan Stone (3), en su catálogo de los mosquitos del mundo la registraron en el subgénero Anoedioporpa Dyar 1.923.

Para la descripción, Rozeboom y Komp utilizaron larvas de esta especie encontradas en internodios de bambú en un lugar del municipio de Acacías, departamento del Meta, Colombia S.A. y a partir de estas larvas desarrollaron las formas adultas. Sin embargo, la pupa de este mosquito no fue descrita. El objetivo de este trabajo es el de dar su descripción a partir de material proveniente de una colonia que el Instituto Nacional de Salud tiene establecida desde el año de 1.983 en un insectario en Armero (Tolima), Colombia S.A.

\section{MATERIAL Y METODOS}

Para la conservación y montaje permanente de las pupas se utilizó, con algunas modificaciones, la técnica de Forattini (4).
Las pupas vivas fueron muertas en agua caliente e inmediatamente se introdujeron en una mezcla de alcohol al $70 \%$ y glicerina, en la proporción de $95 \mathrm{ml}$ de alcohol y $5 \mathrm{ml}$ de glicerina en donde se mantuvieron por espacio de dos meses. Al cabo de los dos meses se procedió a aclararlas y para ello se colocaron en una solución saturada de fenol en alcohol preparada a temperatura ambiente por 24 horas, después de lo cual se pasaron a una solución de potasa $(\mathrm{KOH}$ al 20\%) por 12 horas; posteriormente se deshidrataron en alcoholes ascendentes de $80 \%, 95 \%$ y absoluto por 10 minutos en cada alcohol y finalmente se dejaron en creosota por 24 horas.

Una vez aclaradas las pupas, se procedió al montaje. Para ello con ayuda del microscopio estereoscópico se separaron el abdomen y el cefalotórax; el abdomen se colocó dorsalmente en una gota de bálsamo del Canadá sobre un portaobjeto de vidrio y al cefalotórax se le hizo un corte ventrolongitudinal y se colocó sobre el mismo portaobjeto en posición dorsal.

Pasadas 24 horas, las preparaciones se cubrieron con una laminilla agregando bálsamo del Canadá adicional.

Para el estudio de la quetotaxia de la pupa se utilizó el sistema numérico de Belkin.(5). A las cerdas más importantes se les ha

* Bacteriólogo MSc. Jefe Grupo de Entomología, Instituto Nacional de Salud (INS). Apartado 80334,, Bogotá, Colombia S.A.

** Biólogo. Grupo de Entomología, (INS). 
colocado un asterisco en la descripción, de acuerdo con la técnica de Ramalingam.(6).

Para medir las diferentes estructuras de la pupa se utilizó un microscopio binocular de luz y para elaborar los dibujos se utilizó una cámara lúcida. Las medidas se expresan en milímetros.

\section{DESCRIPCION DE LA PUPA}

Largo total medido desde el cefalotórax al ápice de la paleta $3,08 \mathrm{~mm}$.

El integumento del cefalotórax presenta un color amarillo oscuro en la región del scutum (fig. 1A) y prolongaciones mesotoráxicas y un color amarillo pálido en el integumento del abdomen.

\section{CEFALOTORAX}

La trompeta (fig. 1B) mide $0,38 \mathrm{~mm}$ y presenta un color marrón oscuro, ornamentada con rombos de contornos bien definidos que bordean y sobresalen las áreas del meatus y la reticular, dando la impresión de borde o margen aserrado. El área traqueal es muy reducida y poco aparente. El índice de la trompeta es de 5,77 y la longitud de la pinna es equivalente a 0,038 la longitud de la trompeta.

La quetotaxia del cefalotórax se muestra en la figura $1 \mathrm{C}$.

Cerda $1^{*}$ larga simple muy notable; $2^{*}(1)$ similar a la cerda 1:3 (2) moderadamente larga bifurcada a partir del tercio basal; 4 (3-4), 5(3-2), 6(3-1,2), 7(2), 8(1), 9(1), 10(2-3), $11^{\star}(1), 12^{*}(1-2)$.

\section{ABDOMEN}

El abdomen mide desde el primero al octavo segmento, $2,20 \mathrm{~mm}$.

Presenta microtriquias sobre los segmentos II al VIII. La quetotaxia se muestra en la figura $2 \mathrm{~A}$.

SEGMENTO I: Cerdas 1 (Fig. 2B) de color amarillo claro conformada por 8 a 10 tallos fuertes, subsecuentemente ramificados en 4 a 7 tallos delgados y éstos a su vez presentan cerdas laterales muy finas y dendríticas, $2(1), 3 *(1), 4(4-5,6), 5(1), 6(1), 7(1), 9(1)$.

SEGMENTO II: Cerdas 0(1), 1(10-8,12), 2(1), $3^{*}(1), \quad 4(2-3), 5^{\star}(1), 6(1), 9(1), \quad 10(1)$, $11(1)$,

SEGMENTO III: Cerdas 0(1), 1(3-4), 2(1), $3 *(1), 4(3-2), 5(2-3), 6(1), 7(2-3,4(, 8(2-3,4)$, $11(1), 13(1)$.

SEGMENTO IV: Cerdas 0(1), 1(2-3), 2(1), $3(4-5,6), 4(2), 5^{\star}(1), 6(1), 7(1), 8(2), 10(1-2)$, $11(1)$.

SEGMENTO V: Cerdas O(1), 1(2-1), 2(1), $3(2-3,1), 4(4), \quad 5 *(1-2), \quad 6(1), \quad 7(4-5), \quad 8(2-3)$, $10 *(1), 11(1)$.

SEGMENTO VI: Cerdas 0(1), $1 *(1-2), 2(1)$, $4(2) .5 *(1), 6(1), 10 *(1), 11(1), 8(2), 13(1)$, $14(1)$.

SEGMENTO VII: Cerdas 0(1), 1(1), 2(1), $4(1), 5^{\star}(1), 6(2), 7^{\star}(1), 8(2-3), 9 *(3), 10(2)$, $11(1-2)$.

SEGMENTO VIII: Cerdas O(1), 4(2-3), $9 *(4-3,5)$, con abundantes cerdillas laterales, $14(1)$.

\section{PALETA}

Mide de longitud 0,5 mm. De color amarillo claro, sin pigmentación y no presenta microtriquias; midrib, muy bien estructurado, alargando hasta la porción apical, margen levemente aserrado, sin flequillos.

Cerdas 1,2 presentes por encima del borde apical $(1,5-2,0)$ de la longitud del margen.

El lóbulo genital de la pupa macho, largo, $0,095 \mathrm{~mm}$ de la longitud de la paleta; el lóbulo genital de la pupa hembra corto y redondeado, 0,047 $\mathrm{mm}$ de la longitud de la paleta.

\section{ESPECIMENES EXAMINADOS}

Se examinó un total de 10 pupas hembras y 5 pupas machos. 
ALBERTO MORALES, EMILIO CURA

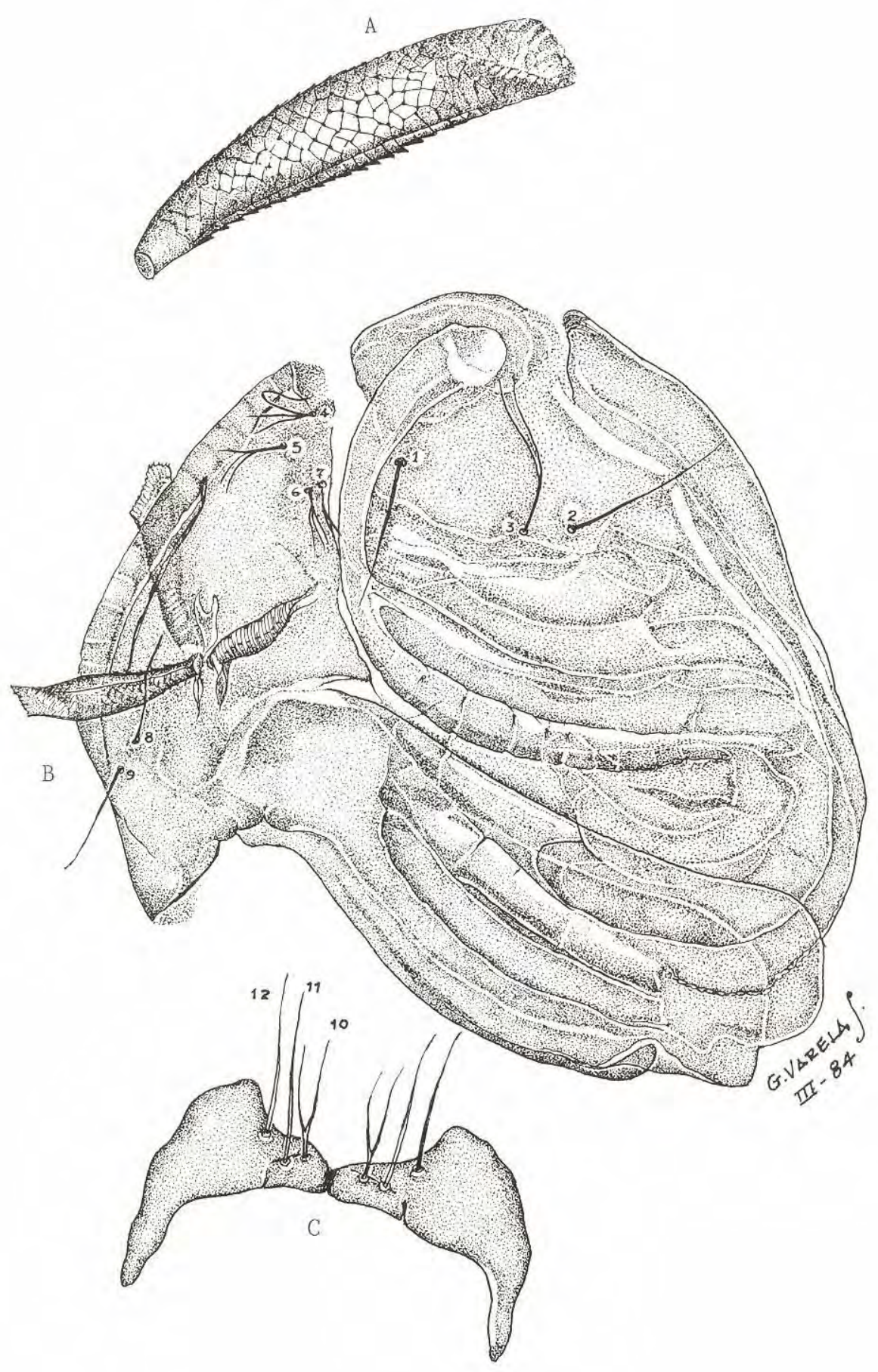

Fig. 1.- $\frac{\text { Culex }}{\text { B-Cefalotórax. C-Scutum }}$ Anoedioporpa $)$ bamborum. Arompeta 
DESCRIPCION DE LA PUPA DE CULEX (ANOEDIOPORPA)....

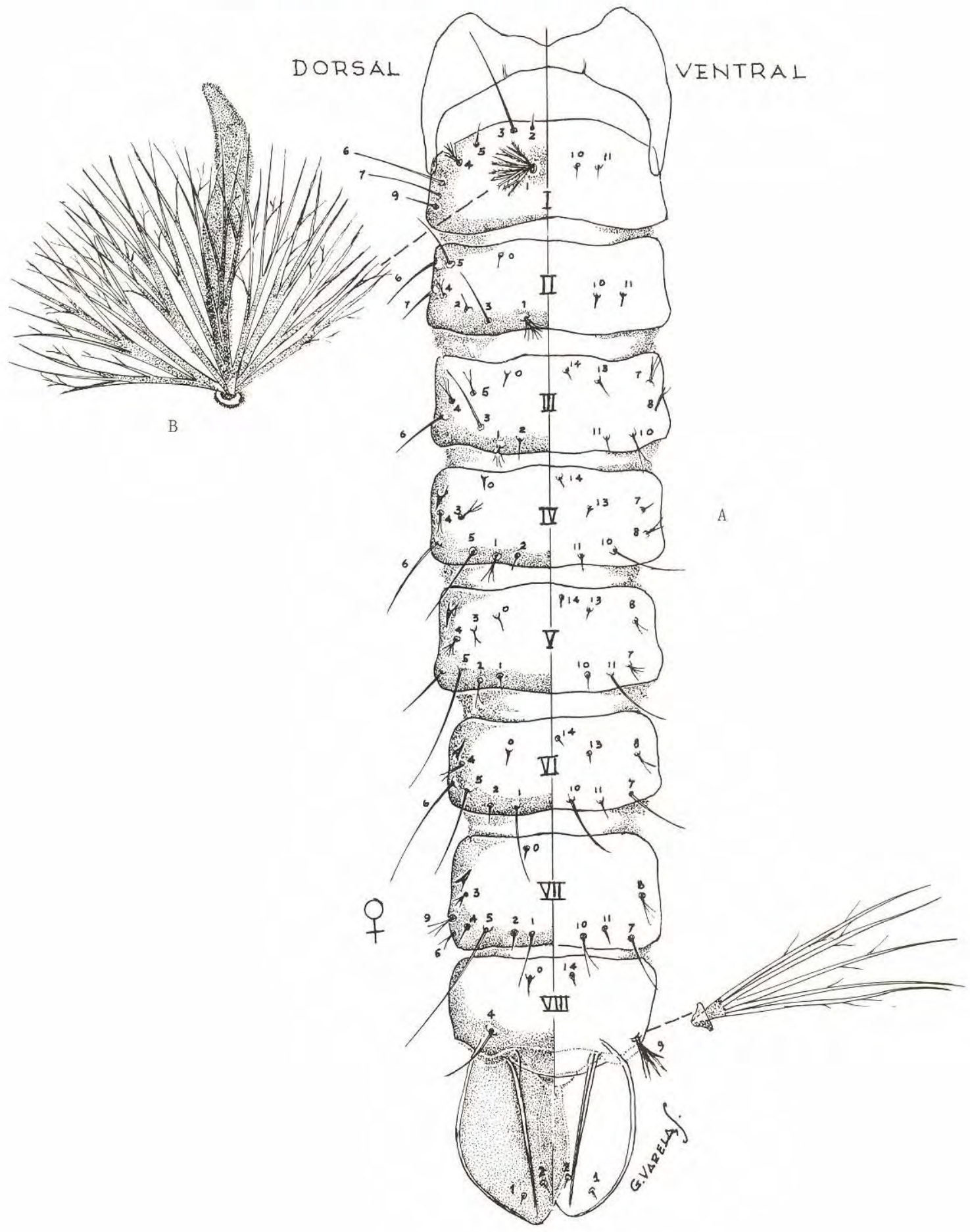

Fig . 2.- Culex ( Anoedioporpa ) bamborum. A-Abdomen.

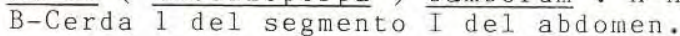




\section{BIBLIOGRAFIA}

The pupa of Culex (Anoedioporpa) bamborum is described out of a colony of the species established in the insectarium of the National Institute of Health (INS) in the town of Armero (Tolima), Colombia, S.A.

\section{AGRADECIMIENTOS}

Los autores desean expresar sus agradecimientos a la Microbióloga Cristina Ferro por su valiosa cooperación en la identificación de la especie. Al señor Eutimio Guerra por el mantenimiento de la colonia. Al señor Guillermo Varela por la elaboración de los dibujos y a la señora Clara Paz de Machado por la transcripción del manuscrito.
1 Rozeboom, L.E. and W.H. Komp. Three new especies of Culex J. Parasit 1948, 34: 396.

2 Belkin, J.N., R.X. Schick, and S.J. Heinemann. Mosquito Studies (Diptera: Culicidae) Mosquitoes originally described from Middle América. Contr. Amer. Ent. Inst, 1965, Vol 1 (5): 1.

3 Knight K.L., Stone A. A catalog of the mosquitoes of the world. Edt. Board., 1.977. Vol VI: 195.

4. Forattini O.P. Entomología Médica Edt. Edanee., 1.962; Vol 1: 614.

5. Belkin, J.N. The mosquitoes of the South Pacific (Diptera: Culicidae) Univ. California 1.962, Vol. 1: 20 .

6. Ramalingam S. Topomyia houghtoni Feng. A new record in Malaysia and redescription of the adult and immature stages. Mosq. Syst 1.983, Vol. $15(1) ; 33$. 